\title{
Preface: DGAA Special Issue on Dynamic Games for Networks
}

\author{
Eitan Altman • Tijani Chahed • Salah-Eddine Elayoubi
}

Published online: 24 January 2013

(C) Springer Science+Business Media New York 2013

This Special Issue of Dynamic Games and Applications (DGAA), entitled Dynamic Games for Networks, contains six papers, having all in common a dynamic game approach to model, investigate, and solve issues as diverse as energy-efficient power control in multiple access channels, a new dynamic formulation of the Hawk and Dove game, design of a resilient encoder capable of transforming monitoring structures, smart grid dynamic demand response management, linear quadratic games with set up costs that are monotonic in the number of active players, typical of joint replenishment inventory systems, as well as interference management in heterogeneous networks, composed of macro and femto cells.

Specifically, the paper by François Mériaux, Samson Lasaulce, and Hamidou Tembine investigates the problem of energy-efficient power control in multiple access channels using a stochastic differential game approach, taking into account long term strategic interactions between players as well as long term energy constraints. The paper by Yezekael Hayel, Veronica Belmega, and Eitan Altman revisits the Hawk and Dove game within a dynamic setting. The authors adopt an evolutionary game approach in which the strategies of the player, and not the standardly used fitness functions, determine the dynamic evolution of the population. In the paper co-authored by Maël Le Treust and Samson Lasaulce, the monitoring structure of dynamic games is considered. The authors specifically address the design of an encoder that is capable of transforming an (arbitrary) monitoring structure by observing the played actions and sending a complementary additional signal to the players through a limited-capacity communication channel.

E. Altman (凶)

INRIA Sophia Antipolis, 2004 route des Lucioles, 06902 Sophia Antipolis Cedex, France e-mail: eitan.altman@inria.fr

T. Chahed

Telecom SudParis, 9 rue Charles Fourier, 91011 Evry Cedex, France

S.-E. Elayoubi

Orange Labs, 38-40 rue du General Leclerc, 92130 Issy-Les-Moulineaux, France 
In the paper by Quanyan Zhu and Tamer Basar, the authors consider dynamic demand response management in smart grids and model the interactions between players, both intragroup and inter-group, for a large population size, within a multi-resolution stochastic differential game-theoretic framework. The paper by Dario Bauso and Raffaele Pesenti investigates linear quadratic games with set up costs that are monotonic in the number of active players, as is the case of joint replenishment inventory systems, under a large population setting, and analyze the impact of an additional disturbance in the framework of H-infinity control. Eventually, the paper by Ana Galindo-Serrano and Lorenza Giupponi considers a heterogeneous network, composed of macro and femto cells, and models the interference management problem using a stochastic game approach; the proposed solution, based on Reinforcement Learning, is optimized so as to maximize the system performance.

To close this editorial, we wish to thank all the people who contributed to the preparation and publication of this special issue. We express our deepest gratitude to the authors for providing us with excellent content and choosing this special issue for the submission and publication of their work. We extend our sincere thanks to the anonymous reviewers for their invaluable support, effort, and time. Our warmest thanks go to the Editor-in-Chief of DGAA, Georges Zaccour, for his continuous guidance, trust and support. A big thank you to the editorial team in Springer for their patience, kindness, and help.

We wish you all to enjoy the reading experience of this special issue as much as we enjoyed editing it.

Bonne lecture!

Editors

E. Altman

T. Chahed

S.-E. Elayoubi 